\title{
Lumped Mass Finite Element Method of the Nonlinear BBM Equation
}

\author{
Hongying Si \\ College of Mathematics and Statistics, Shangqiu Normal University, Shangqiu, China \\ Email: sihongying@126.com
}

How to cite this paper: Si, H.Y. (2021) Lumped Mass Finite Element Method of the Nonlinear BBM Equation. Open Journal of Applied Sciences, 11, 1028-1037. https://doi.org/10.4236/ojapps.2021.119075

Received: August 30, 2021

Accepted: September 19, 2021

Published: September 22, 2021

Copyright (c) 2021 by author(s) and Scientific Research Publishing Inc. This work is licensed under the Creative Commons Attribution International License (CC BY 4.0)

http://creativecommons.org/licenses/by/4.0/

\begin{abstract}
In this paper, the full-discrete approximation scheme of the lumped mass nonconforming finite element method for BBM equation is discussed. Without the Riesz projection used in the traditional finite element analysis, the optimal error estimations are derived based on interpolation technique and special properties of element.
\end{abstract}

\section{Keywords}

Lumped Mass, BBM Equation, Crank-Nicolson Scheme, Nonconforming Finite Element

\section{Introduction}

The lumped mass finite element method is a kind of modified finite element method. It has the same convergence and error estimation as the traditional finite element method, but it has a smaller amount of calculation. Therefore, the lumped mass finite element method is favored by scholars at home and abroad. It is also one of the hot topics being studied [1]-[10]. It is known that equations of this type arise in many areas of mathematical physics and fluid mechanics. It has been studied extensively by Benjamin and others, as a model for unidirectional, long, dispersive waves. It has been widely used in linear optics, iso-particle physics, etc. The numerical solution of problem has been studied in [11] [12] [13] [14], among them, the standard Galerkin method, the finite difference method and the general method are applied to this equation. Feng Minfu et al. proposed a Crank-Nicolson difference method to discretize the equation in [11]. Khaled Omrani made a detailed analysis of the standard Galerkin method of this equation in [14]: the space is discretized by the standard Galerkin, and the time discretization is in the Crank-Nicolson format, the convergence of the me- 
thod is proved.

Tan Yanmei et al. applied the mixed finite element method to this equation in [15], established semi-discretization and full discretization finite element format, the existence and uniqueness of the finite element solution is proved, and an error analysis is given. However, the research on the lumped mass finite element method of nonlinear BBM equations has not been reported.

The main purpose of this article is to study the lumped mass finite element method for the BBM equation, and approximation using non-conforming triangle elements. Through the particularity of unit construction and interpolation techniques, the optimal error estimate is obtained without the need for traditional Reiz projection. In this paper, $\mathrm{C}$ denotes generic positive constant independent of step sizes and not necessarily the same at each occurrence.

\section{Lumped Mass Nonconforming Fully Discrete Crank-Nicolson Scheme of BBM Equation}

We will consider the following nonlinear BBM equation:

$$
\begin{cases}u_{t}-\Delta u_{t}=\nabla f(u), & \forall(X, t) \in \Omega \times(0, T], \\ u(X, t)=0, & \forall(X, t) \in \partial \Omega \times(0, T], \\ u(X, 0)=u_{0}(X), & \forall X \in \bar{\Omega} .\end{cases}
$$

where $\Omega \subset R^{2}$ is a bounded domain with smooth boundary $\partial \Omega, 0<T<\infty$, and $f(u)=-\left(\frac{1}{2} u^{2}+u\right), \quad X=(x, y)$.

For a nonnegative integer $m$, let $H^{m}(\Omega)$ denote the usual Sobolev space of real-valued functions defined on $\Omega$.

$$
H^{m}(\Omega)=\left\{v ; \sum_{0 \leq|\alpha| \leq m} \int_{\Omega}\left|\frac{\partial^{|\alpha|} v}{\partial x^{\alpha_{1}} \partial y^{\alpha_{2}}}\right|_{0}^{2} \mathrm{~d} x \mathrm{~d} y<\infty\right\},
$$

Further, let

$$
H_{0}^{1}(\Omega)=\left\{v \in H^{1}(\Omega), v=0 \text { on } \partial \Omega\right\} .
$$

The norm of this space is the usual Sobolev $H^{m}$-norm and it will be denoted by $\|\cdot\|_{m}$,

$$
\|v\|_{m}=\left[\sum_{0 \leq \alpha \mid \leq m} \int_{\Omega}\left|\frac{\partial^{|\alpha|} v}{\partial x^{\alpha_{1}} \partial y^{\alpha_{2}}}\right|_{0}^{2} \mathrm{~d} x \mathrm{~d} y\right]^{\frac{1}{2}}
$$

and semi-norm

$$
|v|_{m}=\left[\sum_{|\alpha|=m} \int_{\Omega}\left|\frac{\partial^{|\alpha|} v}{\partial x^{\alpha_{1}} \partial y^{\alpha_{2}}}\right|^{2} \mathrm{~d} x \mathrm{~d} y\right]^{\frac{1}{2}},
$$

where, $\alpha=\left(\alpha_{1}, \alpha_{2}\right), \alpha_{1}$ and $\alpha_{2}$ are two non-negative integers $|\alpha|=\alpha_{1}+\alpha_{2}$, specially, let $l=0, m=0$, denoted as $L^{2}(\Omega)=H^{0}(\Omega),(\bullet, \bullet)$ denote the inner 
product in $L^{2}(\Omega)$.

We introduce the weak formulation of (1). Let $u_{t}=Q$ by $v \in H_{0}^{1}(\Omega)$ and use the Green formula.

$$
\left\{\begin{array}{l}
\left(u_{t}, v\right)+a(Q, v)=(\nabla f(u), v), \\
\left(u_{t}, v\right)=(Q, v), \\
u(X, 0)=u_{0}(X) .
\end{array}\right.
$$

where $a(Q, v)=(\nabla Q, \nabla v)=\int_{\Omega} \nabla Q \nabla v \mathrm{~d} X$.

Proposition 1. Let $u$ be the solution of (2). Then, the following conservation of energy holds.

$$
\|u(t)\|_{1}=\|u(0)\|_{1}, \quad \forall t>0 .
$$

Proof. Setting $v=u$ in (2), we get

$$
\frac{1}{2}\left(\frac{\mathrm{d}\|u\|^{2}}{\mathrm{~d} t}+\frac{\mathrm{d}\|\nabla u\|^{2}}{\mathrm{~d} t}\right)=\int_{\Omega} u \nabla f(u) \mathrm{d} X .
$$

Noting that

$$
u \nabla f(u)=\nabla(f(u) u)-\nabla(\varphi(u)), \quad \forall u \in H_{0}^{1}(\Omega)
$$

where $\varphi^{\prime}(u)=f(u)$. Since $u=0$, on $\partial \Omega$ and $\varphi(0)=0$, then

$$
\int_{\Omega} u \nabla(f(u)) \mathrm{d} X=\int_{\Omega} \nabla(f(u) u) \mathrm{d} X=0,
$$

Form (4) and (5). It follows that,

$$
\frac{\mathrm{d}}{\mathrm{d} t}\|u\|_{1}^{2}=0
$$

Integrating (6) with respect to $t$, to complete the rest of the proof.

Let $\Omega \subset R^{2}$ be a polygon on $\left(x_{1}, x_{2}\right)$ plane with boundaries parallel to the axes. $T_{h}$ be an triangle subdivision of $\Omega . \bar{\Omega}=\bigcup_{K \in T_{h}} K$, which does not need to satisfy the regularity assumption or quasi-uniform assumption [6]. For a give $K \in T_{h}$, the three vertices of $K$ are $a_{1}=(0,0), a_{2}=\left(h_{x}, 0\right), a_{3}=\left(0, h_{y}\right)$. Let $\hat{K}$ be the reference element on $(\xi, \eta)$ plane, the three vertices of $\hat{K}$ are $\hat{a}_{1}=(0,0), \hat{a}_{2}=(1,0), \hat{a}_{3}=(0,1)$.

For all $\hat{v} \in H^{1}(\hat{K})$, we define the finite element $(\hat{K}, \hat{P}, \hat{\Sigma})$ on $\hat{K}$ as follows [16]

$$
\hat{\Sigma}=\left\{\hat{v}_{1}, \hat{v}_{2}, \hat{v}_{3}\right\}, \quad \hat{P}=\operatorname{span}\{1, \xi, \eta\}
$$

where $\hat{v}_{i}=\frac{1}{\left|\hat{l}_{i}\right|} \int_{\hat{l}_{i}} \hat{v} \mathrm{~d} \hat{s}, \quad i=1,2,3, \hat{l}_{i}$ represent the edge of $\hat{K}$.

It can be easily checked that interpolation defined above is well-posed and the interpolation function $\hat{\prod} \hat{v}$ can be expressed as

$$
\hat{\Pi}: \frac{1}{\left|\hat{l}_{i}\right|} \int_{\hat{l}_{i}}(\hat{v}-\hat{\Pi} \hat{v}) \mathrm{d} \hat{s}=0, \quad i=1,2,3 .
$$


then

$$
\hat{\Pi} \hat{v}=\left(\hat{v}_{3}+\hat{v}_{1}-\hat{v}_{2}\right)+2\left(\hat{v}_{2}-\hat{v}_{3}\right) \xi+2\left(\hat{v}_{2}-\hat{v}_{1}\right) \eta
$$

Define the affine mapping $\hat{K} \rightarrow K$ by $F_{K}: x=h_{x} \xi, y=h_{y} \eta$.

For all $K \in T_{h}$, then we define the associated finite element space $V_{h}$ and bilinear form $a(\bullet, \bullet)$ as

$$
\left\{\begin{array}{l}
V_{h}=\left\{v|\hat{v}=v|_{K} \circ F_{k} \in \hat{P} \cdot \int_{l}[v] \mathrm{d} s=0, l \subset \partial K\right\}, \\
a_{1 h}(u, v)=\sum_{K} \int_{K} \nabla u \nabla v \mathrm{~d} X=\left(\nabla_{h} u, \nabla_{h} v\right) .
\end{array}\right.
$$

where $[v]$ stands for the jump of $v$ across the edge $l$, if $l$ is an internal edge and it is equal to $v$ itself if $l \subset \partial \Omega$.

For $K \in T_{h}$ the associated finite element interpolation is

$$
\begin{aligned}
& \prod_{h}: H^{1}(\Omega) \rightarrow V_{h}, \\
& \left.\prod_{h} v\right|_{K}=\hat{\Pi} \hat{v} \circ F_{K}^{-1} .
\end{aligned}
$$

For $v \in V_{h}$, we define $\|v\|_{1 h}=\left(\sum_{K \in T_{h}} \int_{K} \nabla v \nabla v \mathrm{~d} x \mathrm{~d} y\right)^{\frac{1}{2}}$. It is easy to see that $\|\cdot\|_{1 h}$ is a norm over $V_{h}$.

Now we consider the following numerical integration format

$$
Q_{K, h}(v)=\frac{1}{3} \operatorname{meas}(K) \cdot \sum_{j=1}^{3} v\left(a_{j, k}\right) \approx \int_{K} v \mathrm{~d} x \mathrm{~d} y,
$$

where $a_{j, k}(j=1,2,3)$ is the three vertices of $K$.

According to the Bramble-Hilbert lemma [2] and (10) is exactly true for the linear polynomial, we get

$$
\left|Q_{K, h}(v)-\int_{K} v \mathrm{~d} x \mathrm{~d} y\right| \leq C h^{2} \sum_{|\alpha|=2}\left\|D^{\alpha} v\right\|_{L^{2}(K)} .
$$

Definition

$$
(u, v)_{h}=\sum_{K \in T_{h}} Q_{K, h}(u v),\|u\|_{h}^{2}=(u, u)_{h}
$$

From (11), we know $\|\cdot\|_{h}$ and $\left\|_{\bullet}\right\|_{0}$ are equivalent in space $V_{h}$, then there exist two constants $C_{1}$ and $C_{2}$, independent of $h$ and $k$ such that

$$
C_{1}\|v\|_{0} \leq\|v\|_{h} \leq C_{2}\|v\|_{0}, \quad \forall v \in V_{h} \text {. }
$$

For any given positive integer $N$, let $k=\frac{T}{N}$ denote the size of the time discretization and $0=t_{0}<t_{1}<\cdots<t_{N}=T, \Delta t=t_{n+1}-t_{n}, \quad n=0,1,2, \cdots, N-1$. The linear function determined by the values of two nodes $u^{h}\left(X, t_{n}\right)$ and $u^{h}\left(X, t_{n+1}\right)$ is an approximate solution of $u(X, t)$, then lumped mass nonconforming fully discrete Crank-Nicolson scheme of (2) lets $v \in V_{h}$, to find $\left(u_{n+1}^{h}, Q_{n+1}^{h}\right)$, such that 


$$
\left\{\begin{array}{l}
\left(u_{n+1}^{h}-u_{n}^{h}, v\right)_{h}+a_{1 h}\left(Q_{n+\frac{1}{2}}^{h}, v\right) \Delta t=\left(\nabla f_{n+\frac{1}{2}}, v\right) \Delta t \\
\left(\frac{u_{n+1}^{h}-u_{n}^{h}}{\Delta t}, v\right)=\left(\frac{Q_{n+1}^{h}+Q_{n}^{h}}{2}, v\right), \\
\left(u_{0}^{h}-\prod_{h} u_{0}, v\right)=0 \\
\left(Q_{0}^{h}-\prod_{h} \varphi, v\right)=0
\end{array}\right.
$$

where $u_{0}^{h}$ is an appropriate approximation to $u_{0}(X), u_{n}=u\left(X, t_{n}\right)$, $u_{n}^{h}=u^{h}\left(X, t_{n}\right), Q_{n}^{h}=Q^{h}\left(X, t_{n}\right), u_{n+\frac{1}{2}}^{h}=\frac{1}{2}\left(u_{n}^{h}+u_{n+1}^{h}\right)$, $\nabla f_{n+\frac{1}{2}}=\frac{1}{2}\left(\nabla f\left(u_{n}\right)+\nabla f\left(u_{n+1}\right)\right)$.

According to the theoretical knowledge of the numerical solution of partial differential equations that problem (14) has a unique solution $\left(u_{n+1}^{h}, Q_{n+1}^{h}\right)$.

\section{Error Estimates}

For simplicity, let $u_{n}=u\left(X, t_{n}\right), Q_{n}=Q\left(X, t_{n}\right), \quad \varepsilon_{h}(u, v)=(u, v)_{h}-(u, v)$, $r_{n}=Q_{n}^{h}-\prod_{h} Q_{n}$,

$$
\begin{gathered}
\rho_{n}=Q_{n}-\prod_{h} Q_{n}, \quad \varepsilon_{n}=u_{n}^{h}-\prod_{h} u_{n}, \quad \eta_{n}=u_{n}-\prod_{h} u_{n}, \\
\left.r\right|_{t=0}=\left.\rho\right|_{t=0}=\left.\varepsilon\right|_{t=0}=\left.\eta\right|_{t=0}=0 .
\end{gathered}
$$

Lemma 3.1. There exists a constant $C>0$ such that for all $u, v \in V_{h}$,

$$
\left|\varepsilon_{h}(u, v)\right| \leq C h^{2}\|u\|_{1 h}\|v\|_{1 h} .
$$

Proof. $\forall u, v \in V_{h}, u$ and $v$ are linear functions on the unit $K$, from (11), we have

$$
\left|Q_{k, h}(u, v)-\int_{k} u v \mathrm{~d} x \mathrm{~d} y\right| \leq C h^{2} \sum_{|\alpha|=2}\left\|D^{\alpha}(u v)\right\|_{L^{2}(K)} \leq C h^{2}\|\nabla u\|_{L^{2}(K)}\|\nabla v\|_{L^{2}(K)} .
$$

summing $\mathrm{k}$ and using Cauchy-Schwarz inequality, we get

$$
\left|\varepsilon_{h}(u, v)\right| \leq C h^{2}\|u\|_{1 h}\|v\|_{1 h} .
$$

Lemma 3.2. [16] Suppose $u \in H_{0}^{1}(\Omega) \cap H^{2}(\Omega)$, then, under the anisotropic meshes, there holds

$$
\left|\sum_{k} \int_{\partial k} \frac{\partial u}{\partial n} v \cdot n d s\right| \leq C h\|u\|_{1 h}\|v\|_{1 h}, \quad \forall v \in V_{h} .
$$

where $n=\left(n_{x}, n_{y}\right)$ is the outward unit vector of $\partial K$, let

$$
\begin{aligned}
E_{n}(v)= & \left(\int_{t_{n}}^{t_{n+1}}\left(f-f_{n+\frac{1}{2}}\right) \mathrm{d} t, \nabla v\right)-a_{1, h}\left(\int_{t_{n}}^{t_{n+1}}\left(Q-Q_{n+\frac{1}{2}}\right) \mathrm{d} t, v\right) \\
& -\int_{t_{n}}^{t_{n+1}} \Gamma_{h}\left(Q_{n+\frac{1}{2}}, v\right) \mathrm{d} t+\varepsilon_{h}\left(u_{n+1}-u_{n}, v\right),
\end{aligned}
$$


where $\Gamma_{h}(u, v)=\sum_{k} \int_{\partial k} \frac{\partial u}{\partial n} v \mathrm{~d} s$.

Lemma 3.3. For all $v \in V_{h}$, the solution $u(X, t), Q(X, t)$ of (2), then

$$
\left(u_{n+1}-u_{n}, v\right)_{h}+a_{1 h}\left(Q_{n+\frac{1}{2}}, v\right) \Delta t=\left(f_{n+\frac{1}{2}}, \nabla v\right) \Delta t+E_{n}(v),
$$

where

$$
\begin{aligned}
\left|E_{n}(v)\right| \leq C & {\left[\left(\int_{t_{n}}^{t_{n+1}}\left\|\frac{\partial^{2} f}{\partial t^{2}}\right\|_{0}^{2} \mathrm{~d} t\right)^{\frac{1}{2}}+\left(\int_{t_{n}}^{t_{n+1}}\left\|\frac{\partial^{2} Q}{\partial t^{2}}\right\|_{1}^{2} \mathrm{~d} t\right)^{\frac{1}{2}}\right](\Delta t)^{\frac{5}{2}}\|v\|_{1 h} } \\
& +C h\left(\int_{t_{n}}^{t_{n+1}}\|Q\|_{2}^{2} \mathrm{~d} t\right)^{\frac{1}{2}}(\Delta t)^{\frac{1}{2}}\|v\|_{1 h}+C h^{2}\left(\int_{t_{n}}^{t_{n+1}}\left\|\frac{\partial u}{\partial t}\right\|_{1}^{2} \mathrm{~d} t\right)^{\frac{1}{2}}(\Delta t)^{\frac{1}{2}}\|v\|_{1 h} .
\end{aligned}
$$

Proof. From (2), for all $v \in V_{h}$,

$$
\left(u_{t}, v\right)-\left(\Delta u_{t}, v\right)=(\nabla f(u), v),
$$

Then using Green's formula we have

$$
\left(u_{t}, v\right)+(\nabla Q, \nabla v)=(\nabla f(u), v)+\Gamma_{h}(Q, v),
$$

from Lemma 3.2, for all $v \in V_{h}$, then

$$
\Gamma_{h}(Q, v) \leq C h\|Q\|_{2}\|v\|_{1 h} .
$$

from (17), integral on both sides for $t_{n} \leq t \leq t_{n+1}$, such that

$$
\left(u_{n+1}-u_{n}, v\right)+a_{1, h}\left(\int_{t_{n}}^{t_{n+1}} Q \mathrm{~d} t, v\right)=\left(\int_{t_{n}}^{t_{n+1}} \nabla f \mathrm{~d} t, v\right)+\int_{t_{n}}^{t_{n+1}} \Gamma_{h}(Q, v) \mathrm{d} t,
$$

thus

$$
\begin{aligned}
& \left(u_{n+1}-u_{n}, v\right)+a_{1, h}\left(Q_{n+\frac{1}{2}}, v\right) \Delta t \\
& =\left(\nabla f_{n+\frac{1}{2}}, v\right) \Delta t+\left(\int_{t_{n}}^{t_{n+1}}\left(f-f_{n+\frac{1}{2}}\right) \mathrm{d} t, \nabla v\right)-a_{1 h}\left(\int_{t_{n}}^{t_{n+1}}\left(Q-Q_{n+\frac{1}{2}}\right) \mathrm{d} t, v\right) \\
& \quad+\int_{t_{n}}^{t_{n+1}} \Gamma_{h}\left(Q_{n+\frac{1}{2}}, v\right) \mathrm{d} t+\varepsilon_{h}\left(u_{n+1}-u_{n}, v\right) .
\end{aligned}
$$

and then

$$
\left(u_{n+1}-u_{n}, v\right)_{h}+a_{1 h}\left(Q_{n+\frac{1}{2}}, v\right) \Delta t=\left(f_{n+\frac{1}{2}}, \nabla v\right) \Delta t+E_{n}(v),
$$

let $P_{1}(t)=\frac{t-t_{n}}{t_{n+1}-t_{n}} f\left(X, t_{n+1}\right)+\frac{t-t_{n+1}}{t_{n}-t_{n+1}} f\left(X, t_{n}\right)$, we have

$$
\int_{t_{n}}^{t_{n+1}} P_{1}(t) \mathrm{d} t=\int_{t_{n}}^{t_{n+1}} f_{n+\frac{1}{2}} \mathrm{~d} t .
$$

from (16), for all $v \in V_{h}$, then $\|v\|_{0} \leq C\|v\|_{1 h}$.

According to the one-dimensional linear interpolation theory and the Cauchy-Schwarz inequality, we get 


$$
\begin{aligned}
\left|\left(\int_{t_{n}}^{t_{n+1}}\left(f-f_{n+\frac{1}{2}}\right) \mathrm{d} t, \nabla v\right)\right| & \leq C\left|\left(\int_{t_{n}}^{t_{n+1}} \frac{\partial^{2} f}{\partial t^{2}}(\Delta t)^{2} \mathrm{~d} t, \nabla v\right)\right| \\
& \leq C\left(\int_{t_{n}}^{t_{n+1}}\left\|\frac{\partial^{2} f}{\partial t^{2}}\right\|_{0}^{2} \mathrm{~d} t\right)^{\frac{1}{2}}(\Delta t)^{\frac{5}{2}}\|v\|_{1 h} .
\end{aligned}
$$

there holds

$$
\begin{gathered}
\left|a_{1 h}\left(\int_{t_{n}}^{t_{n+1}}\left(Q-Q_{n+\frac{1}{2}}\right) \mathrm{d} t, v\right)\right| \leq C\left(\int_{t_{n}}^{t_{n+1}}\left|\frac{\partial^{2} Q}{\partial t^{2}}\right|_{1}^{2} \mathrm{~d} t\right)^{\frac{1}{2}}(\Delta t)^{\frac{5}{2}}\|v\|_{1 h}, \\
\left|\int_{t_{n}}^{t_{n+1}} \Gamma(Q, v) \mathrm{d} t\right| \leq C h\left(\int_{t_{n}}^{t_{n+1}}\|Q\|_{2}^{2} \mathrm{~d} t\right)^{\frac{1}{2}}(\Delta t)^{\frac{1}{2}}\|v\|_{1 h}, \\
\left|\varepsilon_{h}\left(u_{n+1}-u_{n}, v\right)\right| \leq C h^{2}\left\|u_{n+1}-u_{n}\right\|\|v\|_{1 h} \leq C h^{2}\left(\int_{t_{n}}^{t_{n+1}}\left\|\frac{\partial u}{\partial t}\right\|_{1}^{2} \mathrm{~d} t\right)^{\frac{1}{2}}(\Delta t)^{\frac{1}{2}}\|v\|_{1 h},
\end{gathered}
$$

which completes the proof.

Lemma 3.4. There exists a constant $r, \Delta t<r<1$, for all $L$, and $1 \leq L \leq N \quad$ ( $L$ is a positive integer), then there holds

$$
\begin{aligned}
\left\|\varepsilon_{L}\right\|_{h}^{2}+\left\|\nabla r_{L}\right\|_{0}^{2} \leq & C\left[\int_{0}^{T}\left(\left\|\frac{\partial^{2} f}{\partial t^{2}}\right\|_{0}^{2}+\left\|\frac{\partial^{2} u}{\partial t^{2}}\right\|_{1}^{2}+\left|\frac{\partial^{2} Q}{\partial t^{2}}\right|_{1}^{2}+\left\|\frac{\partial^{2} Q}{\partial t^{2}}\right\|_{1}^{2}\right) \mathrm{d} t\right](\Delta t)^{4} \\
& +C h^{2} \int_{0}^{T}\|Q\|_{2}^{2} \mathrm{~d} t+C h^{4} \int_{0}^{T}\left\|\frac{\partial u}{\partial t}\right\|_{1}^{2} \mathrm{~d} t .
\end{aligned}
$$

Proof. Subtracting (15) from the first formula of (14), for all $v \in V_{h}$, we have

$$
\left(u_{n+1}^{h}-u_{n+1}-\left(u_{n}^{h}-u_{n}\right), v\right)+a_{1 h}\left(Q_{n+\frac{1}{2}}^{h}-Q_{n+\frac{1}{2}}, v\right) \Delta t=-E_{n}(v) \text {, }
$$

according to the definition of $\varepsilon_{n}, r_{n+\frac{1}{2}}$ and $\eta_{n}$, from (19), we obtain

$$
\begin{aligned}
& \left(\varepsilon_{n+1}-\varepsilon_{n}, v\right)_{h}+\left(\eta_{n+1}-\eta_{n}, v\right)_{h}+a_{1 h}\left(r_{n+\frac{1}{2}}, v\right) \Delta t \\
& -a_{1 h}\left(Q_{n+\frac{1}{2}}-\prod_{h} Q_{n+\frac{1}{2}}, v\right) \Delta t=-E_{n}(v),
\end{aligned}
$$

by the characteristics of the unit of C-R, then

$$
a_{1 h}\left(Q_{n+\frac{1}{2}}-\prod_{h} Q_{n+\frac{1}{2}}, v\right)=0
$$

further, using the second formula of (14), we get

$$
\frac{\varepsilon_{n+1}-\varepsilon_{n}}{2}=\frac{r_{n+1}-r_{n}}{\Delta t}+\frac{\prod_{h}\left(u_{n+1}-u_{n}\right)}{\Delta t}-\frac{\prod_{h}\left(Q_{n+1}-Q_{n}\right)}{2},
$$

let $v=\varepsilon_{n+1}+\varepsilon_{n}$, substitute the above formula in (19), then 


$$
\begin{aligned}
& \left\|\varepsilon_{n+1}\right\|_{h}^{2}-\left\|\varepsilon_{n}\right\|_{h}^{2}+a_{1 h}\left(r_{n+\frac{1}{2}}, 2\left(r_{n+1}-r_{n}\right)\right) \\
& =-E_{n}\left(\varepsilon_{n+1}+\varepsilon_{n}\right)+\left(\eta_{n+1}-\eta_{n}, \varepsilon_{n+1}+\varepsilon_{n}\right) \\
& \quad-a_{1 h}\left(r_{n+\frac{1}{2}}, 2 \prod_{h}\left(\frac{Q_{n+1}-Q_{n}}{\Delta t}-\frac{u_{n+1}+u_{n}}{2}\right)\right) \Delta t,
\end{aligned}
$$

Now we shall respectively estimate the terms at the right end of the Equation (20), from Lemma 3.3 and Young inequality, we get

$$
\begin{aligned}
\left|E_{n}\left(\varepsilon_{n+1}+\varepsilon_{n}\right)\right| \leq & C\left[\int_{t_{n}}^{t_{n+1}}\left(\left\|\left.\frac{\partial^{2} f}{\partial t^{2}}\right|_{0} ^{2}+\right\| \frac{\partial^{2} u}{\partial t^{2}}\left\|_{1}^{2}+\left|\frac{\partial^{2} Q}{\partial t^{2}}\right|_{1}^{2}+\right\| \frac{\partial^{2} u}{\partial t^{2}} \|_{0}^{2}\right) \mathrm{d} t\right](\Delta t)^{4} \\
& +C h^{2} \int_{t_{n}}^{t_{n+1}}\|Q\|_{2}^{2} \mathrm{~d} t+C h^{4} \int_{t_{n}}^{t_{n+1}}\left\|\frac{\partial u}{\partial t}\right\|_{1}^{2} \mathrm{~d} t+\frac{1}{4}\left\|\varepsilon_{n+1}+\varepsilon_{n}\right\|_{h}^{2} \Delta t .
\end{aligned}
$$

Above the second item, we obtain

$$
\left|\left(\eta_{n+1}-\eta_{n}, \varepsilon_{n+1}+\varepsilon_{n}\right)_{h}\right| \leq C h^{2} \int_{t_{n}}^{t_{n+1}}\left|\frac{\partial u}{\partial t}\right|_{1}^{2} \mathrm{~d} t+\frac{1}{4}\left\|\varepsilon_{n+1}+\varepsilon_{n}\right\|_{h}^{2} \Delta t .
$$

From $\left\|r_{n+\frac{1}{2}}\right\| \leq C\left\|\nabla r_{n+\frac{1}{2}}\right\|_{0}$, the third item is estimated as follows

$$
\begin{aligned}
& \left|a_{1 h}\left(r_{n+\frac{1}{2}}, 2 \prod_{h}\left(\frac{Q_{n+1}-Q_{n}}{\Delta t}-\frac{u_{n+1}+u_{n}}{2}\right)\right) \Delta t\right| \\
& \leq 2\left\|\nabla r_{n+\frac{1}{2}}\right\|\left\|_{0}\right\| \nabla\left(\frac{Q_{n+1}-Q_{n}}{\Delta t}-\frac{u_{n+1}+u_{n}}{2}\right) \|_{0} \Delta t \\
& \leq C\left\|\nabla r_{n+\frac{1}{2}}\right\|\left\|\nabla \int_{t_{n}}^{t_{n+1}}\left(\frac{\partial Q}{\partial t}-u_{n+\frac{1}{2}}\right) \mathrm{d} t\right\|_{0} \\
& \leq \frac{1}{2}\left(\left\|\nabla r_{n+1}\right\|_{0}^{2}+\left\|\nabla r_{n}\right\|_{0}^{2}\right) \Delta t+C\left(\int_{t_{n}}^{t_{n+1}}\left|\frac{\partial^{2} u}{\partial t^{2}}\right|_{1}^{2} \mathrm{~d} t\right)(\Delta t)^{4},
\end{aligned}
$$

Substitute the above estimation results in (20), furthermore, from

$$
\frac{1}{2}\left\|\varepsilon_{n+1}+\varepsilon_{n}\right\|_{h}^{2} \leq\left\|\varepsilon_{n+1}\right\|_{h}^{2}+\left\|\varepsilon_{n}\right\|_{h}^{2},
$$

We have

$$
(1-\Delta t)\left(\left\|\varepsilon_{n+1}\right\|_{h}^{2}+\left\|r_{n+1}\right\|_{h}^{2}\right)-(1+\Delta t)\left(\left\|\varepsilon_{n}\right\|_{h}^{2}+\left\|r_{n}\right\|_{h}^{2}\right) \leq \theta_{n},
$$

where

$$
\begin{aligned}
\theta_{n}= & C\left[\int_{t_{n}}^{t_{n+1}}\left(\left\|\frac{\partial^{2} f}{\partial t^{2}}\right\|_{0}^{2}+\left\|\frac{\partial^{2} u}{\partial t^{2}}\right\|_{1}^{2}+\left|\frac{\partial^{2} Q}{\partial t^{2}}\right|_{1}^{2}+\left\|\frac{\partial^{2} u}{\partial t^{2}}\right\|_{0}^{2}\right) \mathrm{d} t\right](\Delta t)^{4} \\
& +C h^{2} \int_{t_{n}}^{t_{n+1}}\|Q\|_{2}^{2} \mathrm{~d} t+C h^{4} \int_{t_{n}}^{t_{n+1}} \|\left.\frac{\partial u}{\partial t}\right|_{1} ^{2} \mathrm{~d} t+C h^{4} \Delta t .
\end{aligned}
$$

For $0<\frac{1}{1+\Delta t}<1$, so that 


$$
\frac{1-\Delta t}{1+\Delta t}\left(\left\|\varepsilon_{n+1}\right\|_{h}^{2}+\left\|r_{n+1}\right\|_{h}^{2}\right)-\left(\left\|\varepsilon_{n}\right\|_{h}^{2}+\left\|r_{n}\right\|_{h}^{2}\right) \leq \theta_{n}
$$

$\left(\frac{1-\Delta t}{1+\Delta t}\right)^{n}$ multiplied with both sides, then summing up from $n=0$ to $L-1$, we get

$$
\left\|\varepsilon_{L}\right\|_{h}^{2}+\left\|\nabla r_{L}\right\|_{0}^{2} \leq\left(\frac{1+\Delta t}{1-\Delta t}\right)^{L} \sum_{n=0}^{N-1} \theta_{n}
$$

for

$$
\left(\frac{1+\Delta t}{1-\Delta t}\right)^{L} \leq\left(\frac{1+\Delta t}{1-\Delta t}\right)^{N}=\left(1+\frac{2 \Delta t}{1-\Delta t}\right)^{N}<\left(1+\frac{2 \Delta t}{1-r}\right)^{N} \leq \mathrm{e}^{\frac{2 T}{1-r}}
$$

then

$$
\begin{aligned}
\sum_{n=0}^{N-1} \theta_{n} \leq & C\left[\int_{0}^{T}\left(\left\|\frac{\partial^{2} f}{\partial t^{2}}\right\|_{0}^{2}+\left\|\frac{\partial^{2} u}{\partial t^{2}}\right\|_{1}^{2}+\left|\frac{\partial^{2} Q}{\partial t^{2}}\right|_{1}^{2}+\left\|\frac{\partial^{2} Q}{\partial t^{2}}\right\|_{1}^{2}\right) \mathrm{d} t\right](\Delta t)^{4} \\
& +C h^{2} \int_{0}^{T}\|Q\|_{2}^{2} \mathrm{~d} t+C h^{4} \int_{0}^{T}\left\|\frac{\partial u}{\partial t}\right\|_{1}^{2} \mathrm{~d} t
\end{aligned}
$$

Finally, use (23) and (24) to complete the rest of the proof.

Theorem 3.1. let $u(X, t)$ and $Q(X, t)$ be the solutions of (2), suppose $f(u)$ is sufficiently smooth, then there holds

$$
\max _{1 \leq n \leq N}\left\{\left\|u_{n}^{h}-u_{n}\right\|_{0}^{2}+\left\|\nabla\left(Q_{n}^{h}-Q_{n}\right)\right\|_{0}^{2}\right\} \leq C\left(h^{2}+(\Delta t)^{4}\right) .
$$

Proof. Using the definition of $\varepsilon_{n}, \eta_{n}, r_{n}, \rho_{n}$ and the triangle inequality, we get

$$
\begin{gathered}
\left\|u_{n}^{h}-u_{n}\right\|_{0}^{2}=\left\|\varepsilon_{n}-\eta_{n}\right\|_{0}^{2} \leq C\left(\left\|\varepsilon_{n}\right\|_{0}^{2}+\left\|\eta_{n}\right\|_{0}^{2}\right) \leq C\left(\left\|\varepsilon_{n}\right\|_{h}^{2}+\left\|\eta_{n}\right\|_{h}^{2}\right), \\
\left\|\nabla\left(Q_{n}^{h}-Q_{n}\right)\right\|_{0}^{2} \leq\left\|\nabla r_{n}\right\|_{0}^{2}+\left\|\nabla \rho_{n}\right\|_{0}^{2},
\end{gathered}
$$

then using Lemma 3.4 and the interpolation theorem, the proof is completed.

\section{Funding}

Key scientific research projects of colleges and universities in Henan Province (NO: 19A110031).

\section{Conflicts of Interest}

The author declares no conflicts of interest regarding the publication of this paper.

\section{References}

[1] Ushijirna, T. (1977) On the Uniform Convergence for the Lumped Mass Approximation to the Heat Equation. Journal of the Faculty of Science, the University of Tokyo, 24, 471-490.

[2] Nie, Y.Y. and Thomee, V. (1985) A Lumped Mass Finite Element Method with Quadrature for a Nonlinear Problem. IMA Journal of Numerical Analysis, 5, 
371-396. https://doi.org/10.1093/imanum/5.4.371

[3] Chen, C.M. and Thomee, V. (1985) The Lumped Mass Finite Element for a Parabolic Problem. The Journal of the Australian Mathematical Society. Series B, 26, 329-354. https://doi.org/10.1017/S0334270000004549

[4] Thomee, V. (1997) Galerkin Finite Element Methods for Parabolic Problems. Springer-Verlag, Berlin, 239-252. https://doi.org/10.1007/978-3-662-03359-3 15

[5] Dai, P.L. (2001) The Lumped Mass Finite Element Method for the Hyperbolic Equations. Applied Mathematics and Computation, 23, 23-28.

[6] Zhang, F.R. (2011) A Nonconforming Finite Element Method with Crank-Nicolson Full-Discrete Scheme for Viscoelasticity Type Equations. Journal of Henan Normal University (Natural Science), 41, 123-128.

[7] Zhang, F.R. and Song, Y.R. (2012) The Lumped Mass Full Discrete Nonconforming Finite Element Method for Hyperbolic Equation. Journal of Anhui Normal University (Natural Science), 36, 5-10.

[8] Zhang, F.R. (2011) The Lumped Mass Nonconforming Finite Element Method of the Crank-Nicolson Scheme for Nonstationary Stokes Problem. Journal of Shandon Normal University (Natural Science), 46, 33-38.

[9] Liang, H.W., Liu, M.F. and Cao, J.W. (2011) Lumped Mass Finite Element Method for Hyperbolic Equation on Rectangular Mesh. Journal of Henan Normal University (Natural Science), 41, 556-561.

[10] Zhang, F.R. and Si, H.Y. (2015) Lumped Mass Finite Element Method of the Nonlinear Klien-Gordon Equation. Journal of Shanxi Normal University (Natural Science), 38, 453-458.

[11] Li, S.K., Feng, M.F. and Li, S. (2003) Finite Difference Approximate Solutions for the Benjamin-Bona-Mahony Equation. Journal of Sichuan Normal University (Natural Science), 26, 363-365.

[12] Luo, Z.D. and Liu, R.X. (1999) Mixed Finite Element Analysis and Numerical Simulation for Burgers Equation. Journal of Applied Mathematics and Computing, 21, 257-268.

[13] Medeiros, L.A. and Perla, M.G. (1977) Existence and Uniqueness for Periodic Solutions of the Benjamin-Bona-Mahony Equation. SIAM Journal on Mathematical Analysis, 8, 792-799. https://doi.org/10.1137/0508062

[14] Omrani, K. (2006) The Convergence of Fully Discrete Galerkin Approximations for the Benjamin-Bona-Mahony Equation. Applied Mathematics and Computation, 180, 614-621. https://doi.org/10.1016/j.amc.2005.12.046

[15] Tan, Y.M., Kong, H., Luo, D. and Feng, M.F. (2015) A Fully Discrete Mixed Finite Method for the Benjamin-Bona-Mahony Equation. Journal d' Analyse Mathématique, 38, 597-609.

[16] Shi, D.Y. and Guan, H.B. (2008) A Kind of Full-Discrete Nonconforming Finite Element Method for the Parabolic Variational Inequality. Journal d Analyse Mathématique, 31, 90-96. 